\title{
Les multiples rôles de l'ubiquitinylation des protéines
}

Les réactions d'ubiquitinylation représentent une modification covalente post-traductionnelle des protéines, et changent les propriétés des protéines cibles et leur destin cellulaire comme le fait la phosphorylation. L'ubiquitinylation est d'abord et surtout connue pour son rôle dans le guidage des protéines vers une voie de dégradation, mais plusieurs études récemment publiées lui attribuent de nouvelles fonctions inattendues.

L'ubiquitine est une protéine de 76 acides aminés, couplée aux protéines à la suite de l'action concertée de trois enzymes. E1 (ubiquitin-activating enzyme, UBC) active I'ubiquitine en présence d'ATP, pour former une liaison thioester de haute énergie sur la glycine amino-terminale de l'ubiquitine. L'ubiquitine est ensuite transférée à une protéine E2 (ubiquitin-conjugating enzyme) chargée de sa conjugaison sur un groupe $\varepsilon-\mathrm{NH}_{2}$ d'une lysine de la protéine cible, en coordination avec une protéine $\mathrm{E} 3$ (ubiquitin protein-ligase) qui contrôle la spécificité du système en reconnaissant la cible. II existe dans la cellule plusieurs E2 (E2a,E2b,E2c) et la famille des enzymes E3 ne cesse de s'agrandir. La reconnaissance du substrat peut être directe par la séquence primaire de la cible, ou induite après phosphorylation de la cible ou interaction de la cible avec une molécule chaperon. Une chaîne de poly-ubiquitinylation est réalisée par I'addition successive de molécules d'ubiquitine sur la lysine 48 de l'ubiquitine précédente dans la chaîne. La protéine poly-ubiquitinylée est ensuite dégradée par le protéasome, l'ubiquitine libérée étant recyclée (pour revue, voir $[1,2]$ ).

L'ubiquitinylation est une étape importante de la voie d'activation des facteurs de transcription de la famille $\mathrm{m} / \mathrm{s} \mathrm{n}^{\circ} 12$, vol. 17, décembre 2001
NF-kB (m/s 1999, n³, p.419). Dansle cytoplasme, NF- $\mathrm{kB}$ est maintenu inactif par une sous-unité inhibitrice $I \kappa B$, et c'est une réaction d'ubiquitinylation qui conduit à l'activation de NF$\kappa B$ via la dégradation de l'inhibiteur $I \kappa B$. Dans une première étape, l'engagement de nombreux récepteurs de surface comme les récepteurs du TNF (tumor necrosis factor) ou de I'IL-1 (interleukine-1) ou la production de différents stress cellulaires (drogues, radiations, radicaux libres) [3] activent un complexe multi-protéique comprenant deux sérine kinases spécifiques $d^{\prime} \mid \kappa B$ (IKK $\alpha, I K K \beta$ ) [4] qui phosphorylent les sérines 32 et 36 en amino-terminal de $I \kappa B$. Puis, une enzyme E3: $\beta T r C P$ ou $E 3 R S^{\prime k B}$, de la famille SCF (Skp-1-cullin-Fbox/WD repeat) (m/s 1999, n8-9 p.1008), reconnaît I KB phosphorylé sur les deux sérines et attire ensuite, via son domaine Fbox, la sous-unité Skp1. Cette dernière sert d'adaptateur pour les protéines Cullin-1 et ROC1 qui recrutent I'enzyme E2 (UBC5 : ubiquitin conjugating enzyme). L'ubiquitinylation s'effectue sur les lysines 21 et 22 de $\mathrm{I} \kappa \mathrm{B}$, entraînant sa dégradation par le protéasome et libérant NF-kB qui peut alors gagner le noyau [4-6] (figure 1). La séquence reconnue par $E 3 R S^{1 \times B}$ (DpSGXXpS) est retrouvée dans trois autres protéines: cactus, I'équivalent d'I $\kappa B$ chez la drosophile, la $\beta$-caténine et la protéine du VIH Vpu. Après ubiquitinylation, cactus, $\beta$-caténine et la molécule CD4 associée à Vpu sont dégradés.

Une nouvelle fonction de l'ubiquitinylation a été mise en évidence dans la voie d'activation de NF-kB par l'interleukine-1 (IL-1). Le récepteur de I'IL-1 utilise de nombreux adaptateurs, dont l'ubiquitine ligase (E3) TRAF6. TRAF6 interagit avec UBC13 (E2) et une protéine apparentée
Uev1A (E1) qui catalysent sa polyubiquitinylation. De façon intéressante, les chaînes d'ubiquitine se fixent sur la lysine 63 de TRAF6 (et non plus la lysine 48) et sont indispensables à I'activation des IKK [10]. Dans ce modèle, l'inhibition pharmacologique du protéasome n'empêche pas l'activation des IKK, montrant que l'ubiquitinylation d'une protéine n'entraîne pas automatiquement sa dégradation mais peut, au contraire, I'activer. En effet, Ub-TRAF6 interagit avec, et active un complexe contenant la sérine kinase TAK1 et les adaptateurs TAB 1 et TAB2. L'activation de TAK1 pourrait résulter d'un changement conformationnel, ou de l'interaction de TAK1 avec la chaîne d'ubiquitine portée par TRAF6. TAK1 agit ensuite directement sur les IKK pour activer la voie NF-KB et sur MKK6 pour activer la voie JNK [11] (figure 1).

De façon différente, l'ubiquitinylation peut participer à la maturation d'une protéine. C'est le cas pour p105 et p100, les précurseurs de p50

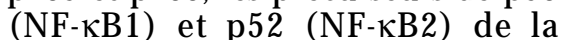
famille NF-kB. P100 et p105 contiennent en carboxy-terminal une région auto-inhibitrice homologue à $\mathrm{I} \kappa \mathrm{B}$. La phosphorylation puis l'ubiquitinylation conduisent à une dégradation partielle de p100 et de p105 qui élimine la région carboxy-terminale inhibitrice, donnant naissance aux facteurs de transcription mûrs p50, p52.

Troisième cible fonctionnelle de l'ubiquitinylation, l'interruption de voies de signalisation. Les récepteurs de facteurs de croissance à activité tyrosine kinase EGFR (récepteur de I'epidermal growth factor) et PDGFR ( récepteur du platelet derived growth factor) sont dégradés après activation par l'intervention de l'enzyme E3 et du proto-oncogène $\mathrm{c}-\mathrm{Cbl}$. Par son 


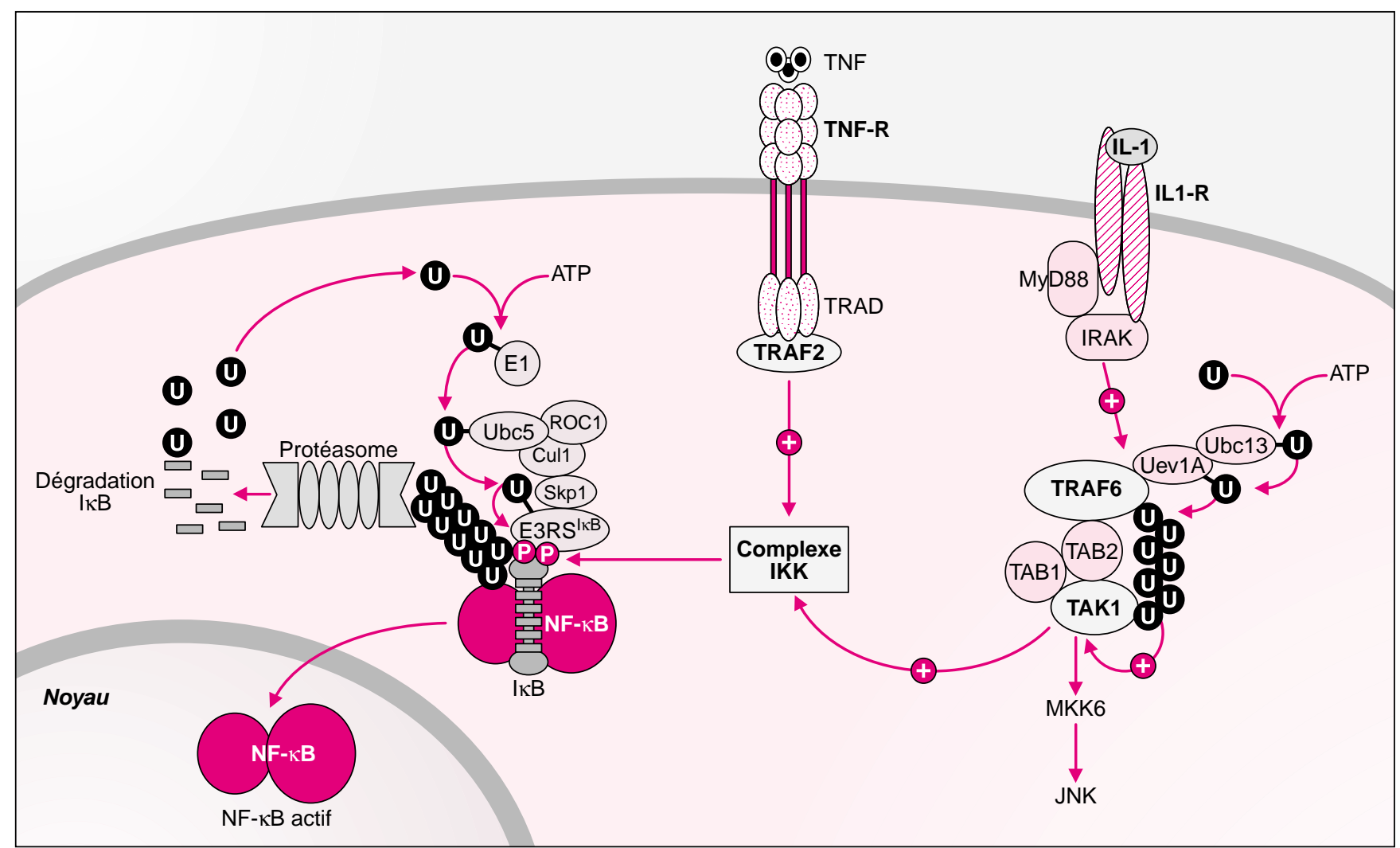

Figure 1. Deux aspects des fonctions de l'ubiquitinylation dans la voie d'activation de NF- $\kappa$ B. Dans la voie de signalisation par le TNF (tumor necrosis factor), I'ubiquitinylation de la sous-unité inhibitrice $\mid \kappa B$, induite après activation du complexe IKK (IKB kinases) et phosphorylation de IKB, conduit à la dégradation de l'inhibiteur et à la libération de $\mathrm{NF}-\kappa \mathrm{B}$ actif. De façon différente et nouvelle, dans la voie de signalisation du récepteur de l'interleukine-1, l'ubiquitinylation de l'adaptateur TRAF6 est cruciale pour la stimulation de la kinase TAK1 qui va mobiliser la voie d'activation de NF- $\kappa$ B ainsi que la voie J NK. D'autres fonctions de l'ubiquitinylation sont discutées dans l'article.

domaine PTB, Cbl reconnaît les phosphotyrosines des récepteurs actifs et recrute une enzyme E2 par son domaine RING. L'ubiquitinylation scelle le sort du récepteur en le détournant vers une voie de dégradation, interrompant ainsi le signal mitogénique [8]. L'ubiquitine participe également au contrôle du cycle cellulaire dans lequel la destruction de certaines cyclines est requise pour la progression dans le cycle (cyclines $A$ et $B$, inhibiteur p27) (pour revue, voir [9]).

L'ubiquitinylation intervient aussi pour dégrader de nombreux facteurs de transcription, mais il existe curieusement une corrélation entre ubiquitinylation et activation de la transcription. Dans un système hétérologue de mesure de la transcription chez la cription VP16 du virus VHS, une enzyme E3 spécifique de VP16 a été identifiée (Met30). L'ubiquitinylation du domaine de transactivation (TAD) de VP16 apparaît importante à la fois pour la dégradation de VP16 et I'activation de la transcription, bien que ces deux fonctions soient dissociables expérimentalement [12]. L'ubiquitinylation pourrait permettre le recrutement de cofacteurs indispensables [12] ou bien permettre le découplage de la polymérase II du complexe d'initiation sur les promoteurs et faciliter ainsi sa fonction d'élongation [13]. Ce mode de contrôle est peut-être opérationnel chez les eucaryotes aussi, et des facteurs comme Myc et Microphtalmia pourraient utiliser I'ubiquitinylation pour moduler leur activité transactivatrice [13].
Dernier exemple de l'intervention de I'ubiquitinylation dans les fonctions cellulaires: le couplage d'une seule ubiquitine sur certaines protéines adaptatrices (Nedd4, Rsp5) est un signal contrôlant de nombreuses étapes du trafic intracellulaire: endocytose, sélection dans le réseau transgolgien et même bourgeonnement viral (pour revue, voir [14]).

Ces différents exemples illustrent de multiples facettes fonctionnelles de I'ubiquitinylation. La conformation des chaînes d'ubiquitine ou le nombre de molécules d'ubiquitine fixées pourraient dicter la spécificité de ces réactions. Le degré de conservation particulièrement important de I'ubiquitine au cours de l'évolution, de la levure à I'homme, renforce I'idée qu'elle contrôle des fonctions cruciales de la cellule. 
1. Ciechanover A. The ubiquitin-proteasome pathway: on protein death and cell life. EMBO J 1998; 17: 7151-60.

2. Laney JD, Hochstrasser $M$. Substrate targeting in the ubiquitin system. Cell 1999; $97: 427-30$. 3. Pahl H. Activators and target genes of Rel/ NF$\kappa \mathrm{B}$ transcription factors. Oncogene 1999; 18: 685366

4. Karin M. How NF-kB is activated: the role of the IkB kinase (IKK) complex. Oncogene 1999; 18 6867-74.

5. Karin M, Ben-Neriah Y. Phosphorylation meets ubiquitinylation : the control of NF-[ kappa]B activity. Annu Rev Immunol 2000; 18: 621-63.

6. Bottero V, Busuttil V, Frelin C, Imbert V, Peyron JF. Les facteurs de transcription NF kappa $B$ : des mécanismes moléculaires d'activation à la régulation de la survie et de la prolifération cellulaires. Regards sur la Biochimie $2001 ; 2$ : 31-9.
7. Palombella VJ, Rando OJ, Goldberg AL, Maniatis $T$. The ubiquitin-proteasome pathway is required for processing the NF-KB1 precursor protein and the activation of NF-kB. Cell 1994; 78: 773-85. 8. Joazeiro C, Wing S, Huang $\mathrm{H}$, Leverson J, H unter T, Liu Y. The tyrosine kinase negative regulator c-Cbl as a RING-type, E2-dependent ubiquitinprotein ligase. Science 1999; 286: 223-5.

9. Koepp D, Harper J, Elledge S. How the cyclin became a cyclin: regulated proteolysis in the cell cycle. Cell 1999; $97: 431-4$.

10. Deng L, Wang C, Spencer E, et al. Activation of the IkB kinase complex by TRAF6 requires a dimeric Ubiquitin-conjugating enzyme complex and a unique polyubiquitin chain. Cell 2000; 103: 351-61.

11. Wang C, Deng L, H ong M, Akkaraju G, Inoue J. Chen Z. Tak1 is a ubiquitin-dependent kinase of MKK and IKK. Nature 2001; 412: 346-51.

12. Salghetti M, Caudy A, Chenoweth J, Tansey W.
Regulation of transcriptional activation domain function by ubiquitin. Science 2001 ; 293: 1651-3. 13. Thomas $D$, Tyers $M$. Transcriptional regulation: kamikaze activators. Curr Biol 2000; 10: 341-3. 14. Hicke LA new ticket for entry into budding vesicles-ubiquitin. Cell 2001; 106: 527-30

\section{Jean-François Peyron}

Inserm U. 526, Faculté de médecine Pasteur, avenue de Valombrose, 06107 Nice Cedex 02, France.

\section{BRÈVES}

Pour le poisson Medaka, un peu de sucre s'il vous plaît. L'importance du poisson zèbre comme modèle anima pour l'étude de la génétique du développement des vertébrés n'est plus à démontrer. Mais le medaka (O ryzias latipes), poisson d'eau douce très prisé des Japonais présente certains avantages par rapport au poisson zèbre: son génome est plus petit, il vit et se reproduit facilement - il peut mêmes'accoupler en apesanteur [1] Depuis plus de trente ans, les mutations se manifestant par des modifications de couleur (environ 70) ont été répertoriées à Nagoya et toutes les données génétiques concernant le medaka peuvent être consultées sur un site: http:// biol1. bio.nagoya-u.ac.jp. Alors que la variété sauvage est brune, la variété la plus recherchée des aquariophiles est rouge orangé. Elle correspond à une mutation au locus $b$ : les mélanophores sont pratiquement invisibles, alors que les autres chromophores sont normalement pigmentés. Un groupe de Japonais vient d'identifier le gène en cause par clonage positionnel. Ce gène, AIM 1 , correspond à un gène humain codant pour un antigène précédemment isolé dans un mélanome (antigen isolated from immunoselected melanoma) [2]. La protéine déduite a 12 domaines transmembranaires. Exprimée tout au long du développement embryonnaire, elle doit être un composant de la membrane des mélanosomes et contribuer au transport de substances nécessaires à la biosynthèse de la mélanine. Fait intéressant, elle porte une séquence d'acides aminés identique à celle des transporteurs de sucrose chez les végétaux, ce qui donne à penser que le transport de sucrose jouerait un rôle dans la synthèse de la mélanine. $O r$, on sait depuis longtemps que les cellules du mélanome murin B6 ont une activité tyrosinase plus élevée dans un milieu contenant du galactose [3], et récemment, il a été montré que les mutations affectant des sites de la $\mathrm{N}$-glycosylation de la tyrosinase réduisent I'activité tyrosinase [4]. I fallait y penser et il faut à présent le vérifier.

[1. Ijiri K. Fish Biol] Medaka 1995; 7 : 48795.]

[2. Fukamachi S, et al. Nat Genet $2001 ; 28$ 381-5.]

[3. Branza-Nichita N, et al. ] BiolChem 2000; 275: 8169-75.]

[4. Saeki H, Oikawa A. J Cell Physiol 1978 94: 139-46.]

Un chaperon pour le VEGF. La production accrue de vascular en dothelia growth factor (VEGF) en réponse à I'hypoxie tissulaire est un des phénomènes majeurs impliqués dans la réponse angiogénique. Ce contrôle de la synthèse de VEGF par la concentration en oxygène des tissus est complexe et fait intervenir une augmentation de la transcription par l'activation du facteur induit par I'hypoxie, HIF, une diminution de la dégradation des ARN du VEGF, et une augmentation de la traduction, inhabituelle en hypoxie, due a la présence sur l'ARNm du VEGF d'un site IRES (internal ribosome entry site). Le VEGF subit ensuite quelques modifications post traductionnelles au cours de son transport intracellulaire et, in fine, est sécrété. Les mécanismes du transport intracellulaire du VEGF sont peu connus et constituent pourtant une cible thérapeutique intéres sante dans la modulation de l'angiogenèse. Une étude récente de Ozawa et al. rapporte que le transport et la sécrétion du VEGF sont contrôlés par une protéine chaperon du réticulum endoplasmique, ORP150 (oxygen regulated protein $150 \mathrm{kDa}$ ) [1]. ORP 150, dont l'expression est contrôlée par l'hypoxie, a été initialement identifiée dans les astrocytes en culture exposés à I'hypoxie. Ozawa et al. montrent que ORP 150 est coexprimée avec le VEGF dans les macrophages du tissu de granulation des lésions cutanées humaines. Chez les souris diabétiques, qui présentent des altérations de la cicatrisation dues à un défaut de vascularisation, I'injection souscutanée de ORP150 insérée dans un vecteur adénoviral au niveau de plaies cutanées accélère les processus de cicatrisation et la formation de néovaisseaux. Dans des macrophages humains en culture exposés à l'hypoxie, l'inhibition de l'expression de ORP150 s'accompagne d'une accumulation de VEGF dans le réticulum endoplas mique tandis que l'augmentation de son expression est associée à une sécrétion accrue de VEGF dans le milieu de culture. Ces résultats indiquent que la présence de ORP150 est nécessaire à la sécrétion de VEGF et que dans les cellules hypoxiques l'augmentation de l'expression de VEGF nécessite une augmentation concomitante de ORP150. En d'autres termes, la stimulation ou l'inhibition d'ORP150 pourraient avoir un effet direct sur l'angiogenèse. Cette hypothèse mérite d'être testée car il pourrait s'agir d'une nouvelle approche thérapeutique dans la prévention et le traitement des maladies ischémiques cardiovasculaires et des tumeurs.

[1. O zawa K, et al. J Clin Invest 2001; 108 : 41-50.] 